\title{
Analysis System for Emotional Behavior in Football (ASEB-F): matches of FC Red Bull Salzburg without supporters during the COVID-19 pandemic
}

\author{
Michael Christian Leitner (iD ${ }^{1,2 凶} \&$ Fabio Richlan (iD ${ }^{1,2 \otimes}$
}

During the COVID-19 pandemic the "Austrian Bundesliga"-as in many other European football leagues-resumed the season around the end of May 2020 without supporters in the stadiums. These so-called "ghost games" represent a unique and unprecedented opportunity to study the effects of the (missing) audience on the behavior and experience of sports professionals. The present study is the first of its kind, aimed at addressing the psychological effects of these "ghost games" on football players, staff, and officials. The newly developed "Analysis System for Emotional Behavior in Football" (ASEB-F) was used to video analyze and compare the behavior of players, staff, and officials in-in sum-20 games of FC Red Bull Salzburg in the "Championship Groups" of season 2018/19 ("regular games") and season 2019/20 ("ghost games"). Additionally, the two seasons were compared based on official matchday statistics. Overall, there were 19.5\% fewer emotional situations in "ghost games" than in "regular games". The results further show a relative increase in the number of emotional behaviors "Self-Adaptor" (+0.8\%), "Protest" $(+4.2 \%)$, and "Fair-Play-Behavior" $(+3.1 \%)$ in "ghost games", whereas "Words fight" (-5.1\%) and "Discussion" (-5.1\%) decreased in "ghost games". In "regular games" referees were actively involved in $39.4 \%$ of all documented emotional situations, whereas in "ghost games" referees were actively involved in only $25.2 \%$ of all documented emotional situations ( $-14.2 \%)$. Chronological analysis within games-from kick-on to kick-off-further shows substantial differences in the temporal occurrence of emotional behavior between "regular games" and "ghost games". The study provides unprecedented insights into the effects of missing supporters in the football games during the COVID-19 pandemic on emotional behavior on the pitch. Without the external factor of supporters, players and staff acted more factually and got less carried away with longer-lasting and extensive "Words fights" and "Discussion". The evidence from this study indicates that-from a sport psychological perspective-the absence of supporters has a substantial influence on the experience and behavior of players, staff, and officials alike.

\footnotetext{
${ }^{1}$ Centre for Cognitive Neuroscience (CCNS), University of Salzburg, Salzburg, Austria. ${ }^{2}$ Department of Psychology, University of Salzburg, Salzburg, Austria.

凶email: michaelchristian.leitner@sbg.ac.at; fabio.richlan@sbg.ac.at
} 


\section{Introduction}

uring the COVID-19 pandemic-on May 12th, 2020-the Austrian government agreed to continue the 2019/ 20 season of the "Austrian Bundesliga". The leaguewhich had been suspended since March 18th, 2020-restarted on June 2nd and was completed 120 games later on July 5th. Thus, the Austrian Bundesliga (UEFA Coefficient Rank: 12) was the 7th European league that continued competition, following in the footsteps of Germany (May 16th), Denmark (May 28th), Ukraine, Poland, Serbia and Hungary (May 30th) ("RAN. Fußball: Die Restart-Termine," 2020).

As in other European leagues, the fundamental requirement to restart the Austrian Bundesliga was to play matches without the presence of supporters; so-called "ghost games". In the days before restarting the Austrian Bundesliga, journalists, (former) players, managers, sport psychologists and fans started hypothesizing about the potential effects of missing supporters on the experience and behavior of football professionals, staff members and officials, and, in turn, on the performance of individual players and teams as a whole. For example, an Austrian sport psychologist, Andreas Marlovits, stated that the biggest challenge for players would be "[...] the attribution of importance $[\ldots]$ " in a "[...] resonance lacking environment [...]" ("DerStandard. Sportpsychologe über Geisterspiele," 2020). Austrian club Wolfsberger AC's coach, Ferdinand Feldhofer, stated that highly competitive players might have problems reaching their top game, missing the crowd's punch; but that younger players might improve due to lack of spectators and less external pressure from the ranks ("DerStandard. Bundesliga-Trainer einig," 2020).

The expected effects of "ghost games" can-in part-be explained within the framework of Allport's performance-related interpretation of Social Facilitation Theory (Allport, 1924) and Zajonc's application of Drive Theory (Zajonc, 1965). Zajonc's theory posits that the presence of others (i.e., the audience in a football arena) increases the players' arousal (or drive) and that this increase, in turn, has an effect on the performance and behavior of the players. Specifically, elevated arousal increases the probability that a player's dominant (or automatic) response occurs. In less complex tasks such as sustaining high energy levels, this increase in arousal is beneficial, whereas in more complex tasks such as inhibiting or controlling emotional reactions, this increase in arousal might be detrimental. In a study on basketball this effect was attributed to differences in interactive behavior between the home and away teams (Varca, 1980). Specifically, the home teams showed more functionally aggressive behavior (e.g., steals, blocked shots, rebounds), whereas the away teams showed more dysfunctionally aggressive behavior (e.g., fouls).

Not only the behavior of players and staff is influenced by the presence of supporters in sport events. A study on football (soccer) games-that were played without spectators following safety requirements after hooligan incidents in Sicily-showed that home teams are favored by officials' decisions during matches (Pettersson-Lidbom and Priks, 2010). Similarly, an experimental study investigated the effects of crowd noise on refereeing decisions in football, concluding that referees "viewing the challenges with background crowd noise were more uncertain in their decision making and awarded significantly fewer fouls (15.5\%) against the home team, compared with those watching in silence" (Nevill et al., 2002, p. 261). It was further shown that the crowd size has a direct effect on the number of first yellow cards awarded to the away team in Cup final games (Downward and Jones, 2007) and on referees' overall decisions (Unkelbach and Memmert, 2010).

Sometimes-especially when the levels of expectation and perceived pressure from the ranks rise, such as during play-offs or championship games-the crowd can exert a negative effect on the performance of the home team (Baumeister, 1984; Carron et al., 2005). It was hypothesized that in this case the home crowd leads to increased self-consciousness of the players, disrupting their (otherwise) automatic skill execution. In this context, it has also been shown that effortful self-regulation impairs athletic performance (Wagstaff, 2014). Little is known, however, on moderating effects on the home (dis)advantage, such as unfamiliarity of playing conditions. Thus, the COVID-19-related "ghost games" represent a unique opportunity to study the effects of the (missing) audience on the behavior and experience of players.

The present study investigates the emotional behavior and experience of professional football players in "ghost games" by (1) introducing the newly developed "Analysis System for Emotional Behavior in Football” (ASEB-F), (2) studying and documenting players' nonverbal behavior during competition and (3) analyzing and interpreting corresponding official statistical data. As a case study, this analysis is applied to the 20 games (in total) of FC Red Bull Salzburg in the "Championship Groups" of season 2018/19 ("regular games") and season 2019/20 ("ghost games"). Owing to the absence of supporters and the associated lack of external stimulation, we expected more "factual" games with less emotional behavior in "ghost games" than in regular matches with supporters.

\section{Methods}

In the Austrian Bundesliga 12 teams play in a first, "basic" phase -against each opponent twice in home and away matches. After 22 rounds, the resulting league table is split into two separate groups and the second, "final" phase of the competition starts: the first 6 teams play against each other for the championship ("championship group"), whereas the last 6 teams play for relegation and one spot in the "UEFA Europa League" ("qualification group"), resulting in another 10 rounds to play. Owing to the COVID-19 pandemic, the league was suspended on March 18th, 2020; right before the start of the Austrian Bundesliga's "final" phase. Therefore, we decided to compare the emotional behavior and experience of players, team staff and officials between matches of the championship group of 2018/19 (with supporters) with the championship group of 2019/20 (without supporters).

Sample. As FC Red Bull Salzburg (RBS) won the championship in 2019 and was again the title favorite for 2020 (note: ultimately RBS won the league again), we decided to analyze this team's matches in detail for potential behavioral conspicuousness during the "ghost games". In order to analyze the players' behavior, the TV broadcasting rights holder of the Austrian Bundesliga-Sky Austria-kindly provided us with original match footage for both seasons. This means that a total of 20 matches of RBS were analyzed in full detail. In order to even further analyze all matches of the complete championship groups, the official stats company of the Austrian Bundesliga-Stats Perform-generously supported us with matchday analytics and data from season 2018/19 and $2019 / 20$.

Test statistics. Kolmogorov-Smirnov tests showed normal distribution of all parameters. Thus, for all statistical analyses, twosided independent samples $t$-tests were used to investigate potential differences between seasons 2018/19 ( $n=10$ games) and 2019/20 ( $n=10$ games). 95\% confidence intervals (CI) are provided with each test statistic. Effect sizes are reported in Pearson's $r$ and calculated as follows: $r=\sqrt{\frac{t^{2}}{t^{2}+\mathrm{df}}}$ 


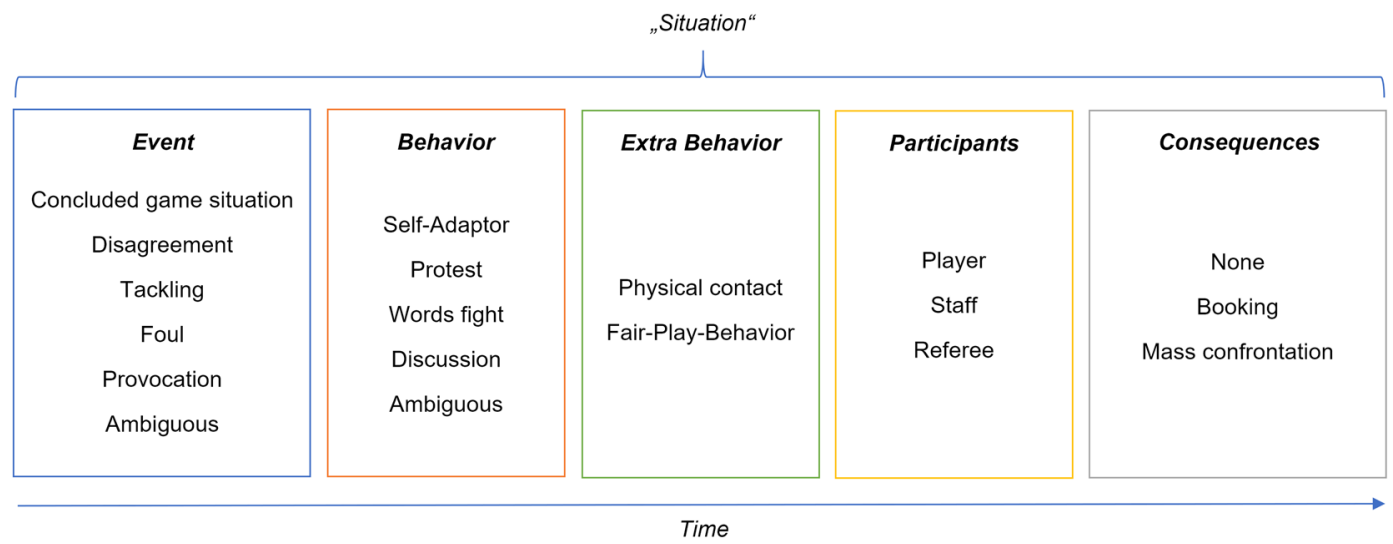

Fig. 1 "Analysis System for Emotional Behavior in Football" (ASEB-F). The system consists of consecutive categories that we developed for this study. The system provides timing for analysis of situations, which are used to describe the initial trigger for the situation itself (event), the resulting visually observable nonverbal reaction (behavior \& extra behavior), involved agents (participants) and the outcome (consequences). One factor from each of the categories "Event", "Behavior" and "Consequences" and at least one factor from the categories "Extra Behavior" and "Participants" must be existent for the situation to qualify for documentation during the analysis of a particular match.

Operationalization. Originating from the work of Richard Lazarus, Michael Eaves, and Dale G. Leathers we created a categorical analysis system with the goal to identify, classify and document visually observable behavior of football players, staff and officials. The "Analysis System for Emotional Behavior in Football" (ASEB-F) is based on the assumption that emotions can be described as an organized psychophysiological reaction to specific events in the environment, rising to overt actions or impulses, leading to human behavior and ultimately resulting in the pursuit of a related goal (Lazarus, 2000). Therefore, overt behavior is visually displayed in nonverbal actions like mimic, gesture, body contact or spatial behavior (Eaves and Leathers, 2018). Specifically, observable behavior illustrates players', staffs' and officials' emotionally (aroused) state due to a situational cause during particular events in a football match. Categorizing those nonverbal cues enables the attribution of different levels of emotional states and experiences and documenting potential differences between regular matches and "ghost games". Therefore, "[...] nonverbal communication is our richest source of knowledge about emotional states [...] and that nonverbal cues are reliable and stable indicators of the emotion that is being conveyed or received." (Eaves and Leathers, 2018, p. 7).

As illustrated in Fig. 1, our five categories of ASEB-F reflect the conceptual approach to the emergence and expression of emotions stemming from interpersonal situations. We understand a football match as a series of those consecutive situations, in which specific behavior can be observed and described, based on the following categories: "Event" stands for specific events in the environment, which lead to "Behavior" and "Extra Behavior" representing psychophysiological reactions that rise to overt actions or impulses. The category "Participants" documents involved stakeholders and "Consequences" describes related implications.

The "Event" category is divided into six distinct factors, while only one factor per documented situation should be selected.

a. "Concluded game situation": The game is interrupted after a shot or header with the ball out of bounds or safe with the goalkeeper.

b. "Disagreement": The game is interrupted due to offside or any other potential disputatious situation.

c. "Tackling": The game is interrupted after a fair tackling (e.g., ball out of bounds).

d. "Foul": The game is interrupted due to a foul (foul is given by official). e. "Provocation": The game is interrupted due to provocative behavior of a player/staff member or the provocative behavior happens during a game interruption.

f. "Ambiguous": any event not applicable to above factors.

The "Behavior" category is divided into five distinct factors, while only one factor per documented situation should be selected.

a. "Self-Adaptor": undirected nonverbal behavior (e.g., selfreproaches after missed chance).

b. "Protest": directed nonverbal behavior with no or very little response by other participant(s) (e.g., players protest after conceded foul and referee shaking his head slightly in response).

c. "Words Fight": directed verbal and nonverbal behavior with clear reaction by other participant(s) (e.g., players protest after conceded foul and referee responding with brief but clear verbal and/or nonverbal reaction).

d. "Discussion": directed verbal and nonverbal behavior with clear and turn-take-reactions between participants (e.g., players protest after conceded foul resulting in back and forth argumentation with referee).

e. "Ambiguous": any behavior not applicable to above factors.

The "Extra Behavior" category is divided into two distinct factors. None, one, or both factors can be selected per documented situation.

a. "Physical Contact": visually observable aggressive physical contact in the course of documented behavior between participants (e.g., player pushing opponent player during discussion after foul).

b. "Fair-Play-Behavior": Good sportsmanship-like behavior (e.g., player helping opponent player up after tackling).

The "Participants" category is divided into three distinct factors. At least one factor should be selected per documented situation.

a. "Player": At least one player of one team is involved in the documented situation.

b. "Staff": At least one staff member (e.g., coach) is involved in the documented situation.

c. "Referee": At least one official (e.g., head-referee) is involved in the documented situation. 
The "Consequences" category is divided into three distinct factors. At least one factor should be selected per documented situation.

a. "None": No booking or mass confrontation in situation.

b. "Booking": At least one player or staff member is booked by referee.

c. "Mass confrontation": $<5$ players from at least one team discussing and interacting in close proximity.

Prerequisites. A match situation only qualifies for documentation during the video analysis-as explained above-when specific characteristics apply:

1. Match interrupted, ball out of play or situation clear and noticeable in the focus of players and/or staff (e.g., players protest while goalkeeper with ball in hands (game not interrupted) waiting for situation to clear up).

2. Some part of the critical situation in "close up" camera shot or situation in another way clear and noticeable in the focus of current game events.

3. Focus of analysis on participants spatially close and actively engaging into the critical situation or specifically shown by "close up" camera shots.

4. No speculation on situations (what might have happened) when not clearly visible (e.g., TV replay overlaps live events).

5. Outbursting behavior lasting shorter than $1 \mathrm{~s}$ are defined as "Macroreactions" or "Microexpressions" and therefore do not qualify for documentation.

6. Football- and sport-specific gestures and communication representing tactical behavior or rules of conduct do not qualify as "emotional behavior" (e.g., coach at the sideline advising players to tackle more fiercely).

\section{Results}

Table position, points, and discipline. A basic prerequisite when comparing a football club's two consecutive seasons is table position and development of points. Analysis of those two factors shows that RBS was continuously positioned at number 1 in the table during both championship group phases of seasons 18/19 and 19/20. In addition, the club's points lead to second place was similar in both seasons and the points lead during 10 games from round 23 to 32 increased similarly ( +8 points in both seasons). The apparent difference between the two seasons was the absolute number of points (18/19: 12 points; 19/20: 9 points) and a dip in round 28 of the 19/20 season. Thus, the linear increase of both curves is evident (season 18/19: $y=0.86^{*} x+4.00, R^{2}=0.75$; season 19/20: $\left.y=0.68^{*} x+2.47, R^{2}=0.71\right)$.

A further crucial factor for comparing players', team staffs' and officials' emotional behavior of matches with and without supporters is the overall team discipline. The Austrian Bundesliga's official statistics provider, Stats Perform, calculates each team's "Disciplinary Points" value by adding 1 point per foul, 3 points per yellow card or yellow/red card, and 6 points per red card. Comparing RBS's championship group Disciplinary Points of season $18 / 19$ (188 points) with season $19 / 20$ (182 points) shows that the values differ by only 6 points $(-3.2 \%)$. Similarly, the opponent teams' discipline was comparable across both seasons, with 222 Disciplinary Points in $18 / 19$ and 206 Disciplinary Points in 19/20, leading to a difference of only 16 points (-7.2\%). Analyzing all 120 games of the two championship group phases (60 games per season), shows that overall fewer fouls were committed $(-3.8 \%)$ and Disciplinary Points were reduced $(-13.5 \%)$ in "ghost games". Temporal course of
"Disciplinary Points" of RBS shows that during the first 6 games of the 18/19 championship group phase, RBS received more Disciplinary Points than during the same rounds of season 19/20. This relation was reversed in the remaining 4 games of the season. Overall, there was no statistically significant difference in disciplinary points of RBS between the two seasons ( $t$ $(18)=0.221,95 \%$ CI $[-5.112,6.312], p=0.828, r=0.052)$.

Fouls and goals. When comparing the two championship group phases of 2018/19 and 2019/20, there were also remarkable differences. When reducing team discipline to "fouls committed" the data shows that RBS committed $13.4 \%$ more fouls in "ghost games" (152 fouls) than in regular games the season before (134 fouls $)(t(18)=-1.167,95 \%$ CI $[-5.040,1.440], p=0.258$, $r=0.265$ ). In contrast, opposing teams fouled less in "ghost games" against RBS (134 fouls) than the season before (141 fouls), leading to a difference of $-5 \%$ in committed fouls in "ghost games" $(t(18)=0.383,95 \%$ CI $[-3.144,4.544], p=0.707$, $r=0.090)$. The resulting similarity in the category Disciplinary Points (see above) can be explained by distinctly fewer yellow, yellow/red and red cards shown to RBS players in "ghost games" $(-41.2 \%)$.

Another major difference can be found in the category "Goals scored". While in 2018/19 RBS scored 28 goals in 10 matches, in 2019/20 RBS scored 36 goals in 10 matches during the championship group phase. This is an increase of 8 goals (6.1\%) and corresponds to a small to medium effect size $(t(18)=$ $-0.894,95 \%$ CI $[-2.679,1.079], p=0.383, r=0.206)$. This trend can also be observed when comparing all 120 games of the two championship group phases (60 games per season). Statistical data shows that in "ghost games" $20 \%$ more goals were scored (114 goals) than in games with regular attendance the year before (95 goals)

Transfers. In the free period between season 2018/19 and 2019/ 20, RBS transferred seven key players (Dabbur, Schlager, Lainer, Samassékou, Wolf, Gulbrandsen, Leitgeb) to other football clubs while transferring six key players (Okafor, Wöber, Kristensen, Adeyemi, Koita, Camara) from other football clubs. These changes in team structure must be considered when comparing the emotional behavior of an entire team between two seasons. As we documented players' jersey numbers in each emotional situation accordingly, we can analyze whether overall participation of RBS players in emotional situations changed between seasons. Figure 2 illustrates relative numbers of involvement of RBS players in emotional situations. We define "high involvement players" as players who are involved in $>6.9 \%$ of all documented emotional situations in season $18 / 19$ and in more than $6.4 \%$ of all documented emotional situations in season 19/20. These two cutoff values represent $50 \%$ of the total involvement of the "highest involvement player" per season (13.7\% for number 9 (Dabbur) in 18/19 and 12.7\% for number 9 (Hwang) in 19/20). Thus, 5 players (9 (Dabbur), 16 (Junuzovic), 21 (Gulbrandsen), 22 (Lainer) and 42 (Schlager)) can be classified as "high involvement players" in $18 / 19$, while player $9(13.7 \%)$ and player 42 $(11.3 \%)$ were more involved in emotional situations than any other player in 19/20. In contrast, 7 "high involvement players"; 5 (Vallci), 9 (Hwang), 14 (Szoboszlai), 16 (Junuzovic), 19 (Camara), 20 (Daka) and 45 (Mwepu) can be documented for season 19/20.

In addition to changes in the team squad, there was a transition in the coaching staff between the two seasons. In particular and most importantly, a new head coach (Jesse Marsch) took over from the former head coach (Marco Rose) for the 2019/20 season. Implications related to this fact are discussed in the Limitations section (see Discussion). 
Championship Group: FC Red Bull Salzburg players involvement in emotional situations

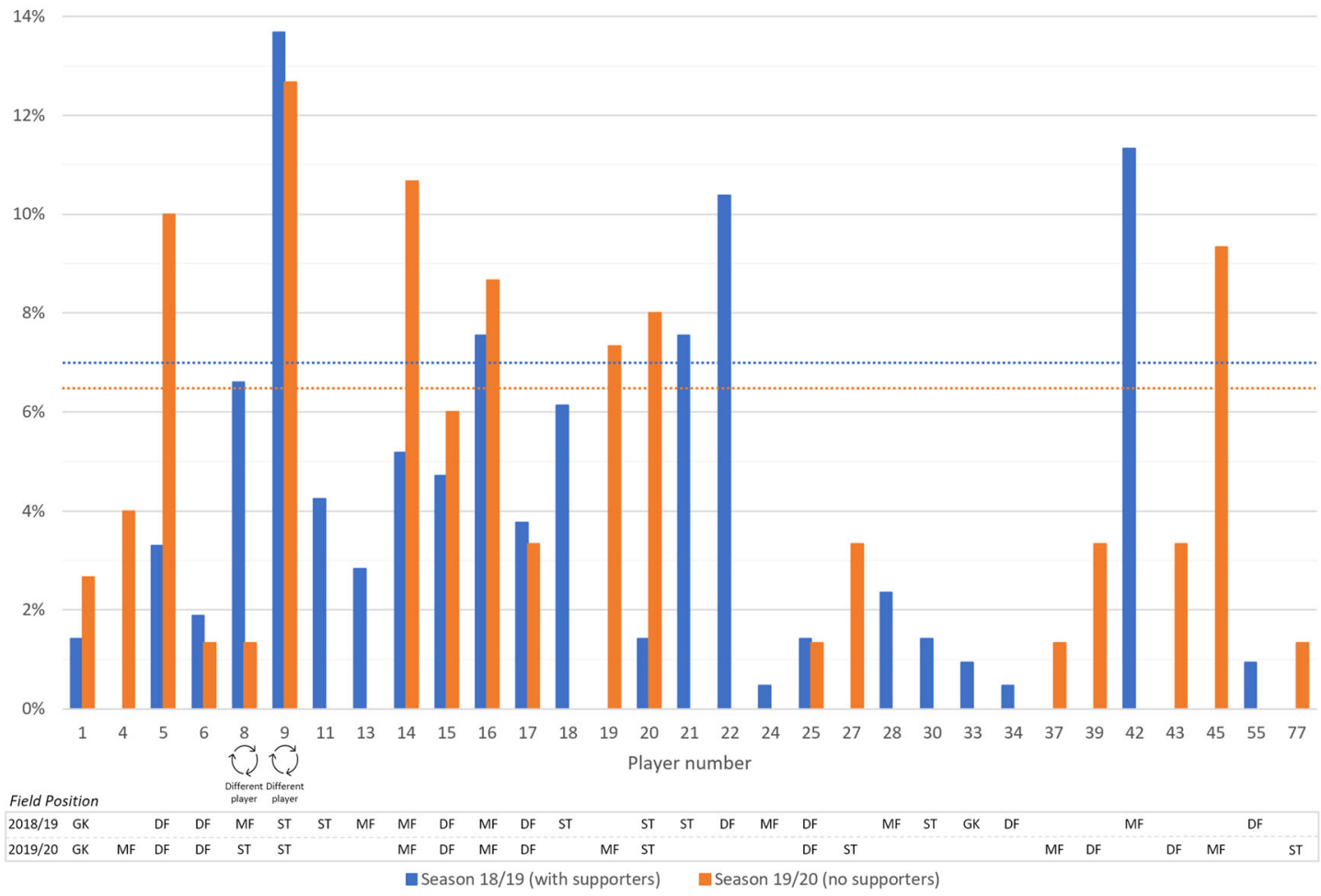

Fig. 2 FC Red Bull Salzburg players involvement in emotional situations. Relative number of involvements of FC Red Bull Salzburg (RBS) players in documented emotional situations of 20 matches in the championship group phases of season 2018/19 and 2019/20. Results indicate that in 18/19, five players can be identified as "high involvement players". We define "high involvement players" as players who are documented more than half of the most involved player per season. That is more than $6.9 \%$ (blue dotted line) for season 18/19 (with number 9 (Dabbur) involved in 13.7\% of all situations) and $6.4 \%$ (orange dotted line) for season 19/20 (with number 9 (Hwang) involved in $12.7 \%$ of all situations). Additional information is provided by illustration of players' field position in respective seasons (GK goalkeeper, DF defender, MF midfielder, ST striker). Except for number 8-which was worn by two different players-all players kept their field position role in both seasons. Thus, in season 18/19 there are 5 "high involvement players": 9 (Dabbur) (ST), 16 (Junuzovic) (MF), 21 (Gulbrandsen) (ST), 22 (Lainer) (DF) and 42 (Schlager) (MF). Two players (9 \& 42) had more involvements than any other player in season 19/20. In season 19/20 there are 7 "high involvement players": 5 (Vallci) (DF), 9 (Hwang) (ST), 14 (Szoboszlai) (MF), 16 (Junuzovic) (MF), 19 (Camara) (MF), 20 (Daka) (ST) and 45 (Mwepu) (MF). Our data suggests that the relative number of interactions of RBS players in emotional situations did not change remarkably due to player transfers between seasons. Additionally, the roles (in terms of field position) of the high involvement players are comparable between both seasons. While in season 2018/19 one defender, two midfielders and two strikers are defined as "high involvement players", in season 2019/20 one defender, four midfielders and two strikers are defined as "high involvement players". This similar distribution indicates that hierarchical and personalities structure in the various parts of the team is comparable between both seasons. Note that two numbers were worn by two different players in season 18/19 and 19/20. As illustrated with small arrows, these are number 8 (Samassékou \& Berisha) and number 9 (Dabbur \& Hwang).

Emotional situations. Our video analysis of RBS games in 20 championship group matches in season 18/19 (10 matches) and $19 / 20$ (10 matches) shows that there are-in total- $19.5 \%$ fewer emotional situations in "ghost games" (season 19/20). This means that-based on our developed "Analysis System for Emotional Behavior in Football” (ASEB-F) - our data shows that players and team staff tend to involve themselves less actively and emotionally in behavior such as "Words fight" or "Discussion" with opponent players and/or referees, corresponding to a medium to large effect size $(t(18)=2.022,95 \%$ CI $[-0.191,9.991], p=0.058, r=0.430)$

Analysis of the duration of all emotional situations shows a similar result, with an akin distribution over time from round 23 to 32. In matches with supporters, players, staff and officials spent in total 41:42 min for emotional behavior, whereas in "ghost games" in total only 27:09 min were documented as "SelfAdaptor", "Protest", "Words fight", "Discussion" or "Fair-PlayBehavior". The shorter duration of emotional situations in "ghost games" compared to games with supporters was statistically significant with a large effect size $(t(18)=2.477,95 \%$ CI $[13.268$, 161.332], $p=0.023, r=0.504)$.

Owing to the conspicuously different total number and duration of overall "Emotional situations" between season 18/19 and 19/20, we calculated these distinct emotional behaviors relative to absolute number-as illustrated in Fig. 3 - and relative to total duration-as illustrated in Fig. 4. Results show that in both relative number and relative duration of emotional behavior, "Self-Adaptor", "Protest", and "Fair-Play-Behavior" increased in "ghost games", whereas the relative duration of "Words fight" and "Discussion" decreased in "ghost games".

Referees and officials. In the ten matches of RBS in both seasons, nine different head referees officiated the games. One referee in both seasons officiated two games respectively, Alexander Harkam in 2018/19 and Robert Schörgenhofer in 2019/20. Five Referees officiated games of RBS in both seasons: Julian Weinberger, Andreas Heiß, Rene Eisner, Harald Lechner and Josef 
Championship Group: Relative number of distinct "Emotional situations" in matches with FC Red Bull Salzburg

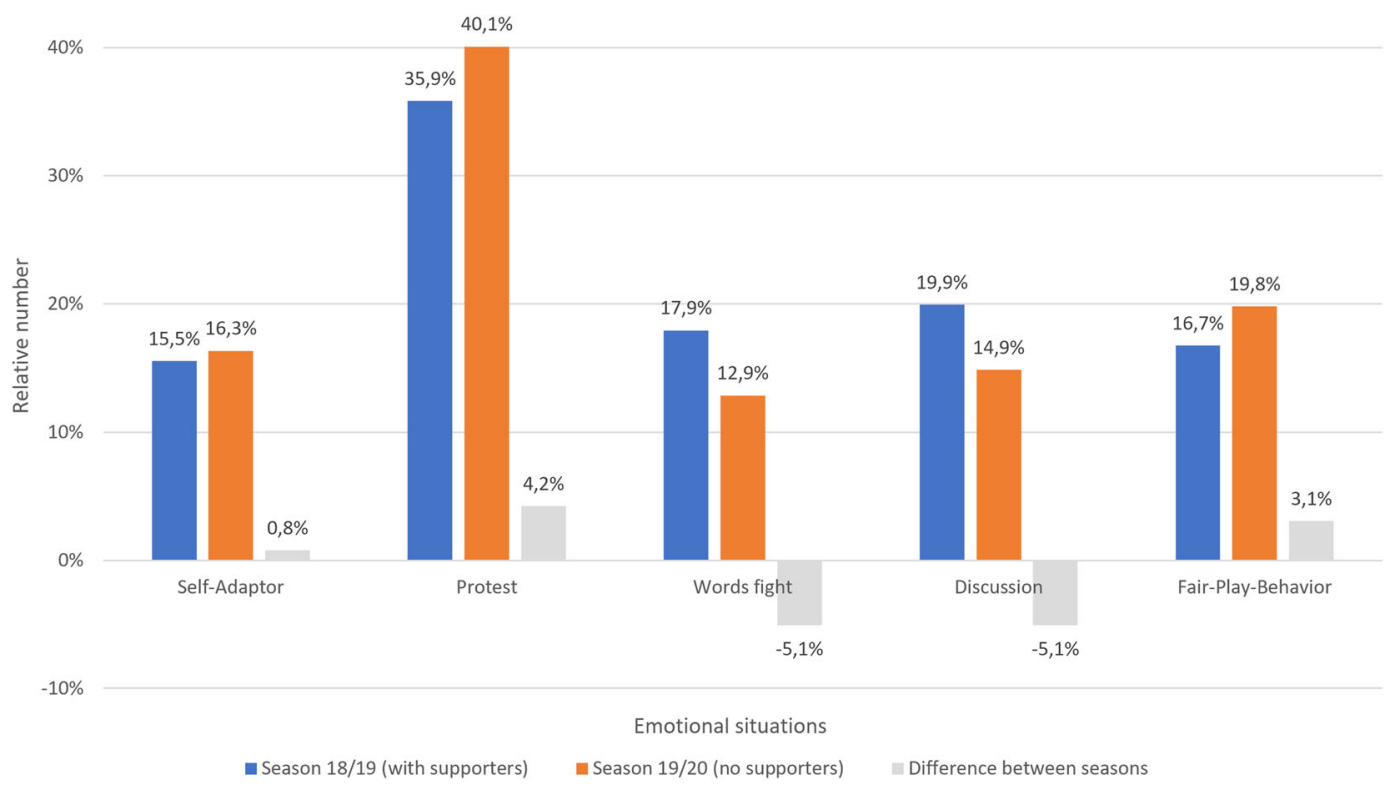

Fig. 3 Relative number of "Emotional situations". Differences in the number of distinct emotional behavior of players, staff, and officials in matches with FC Red Bull Salzburg (RBS) during the championship group phase in both seasons of 2018/19 and 2019/20 relative to the overall number of emotional situations. Video documentation and analysis is based on our developed "Analysis System for Emotional Behavior in Football" (ASEB-F) (see above for details). Data shows the number of emotional behaviors "Self-Adaptor", "Protest", and "Fair-Play-Behavior" increased in "ghost games", whereas "Words fight" and "Discussion" decreased in "ghost games". This indicates that while players, staff and officials acted predominantly less emotionally in "ghost games", "less extensive" behavior like "Self-Adaptor", "Protest", and "Fair-Play-Behavior" increased in number and "more extensive" behavior like "Words fight" and "Discussion" decreased in duration in "ghost games".

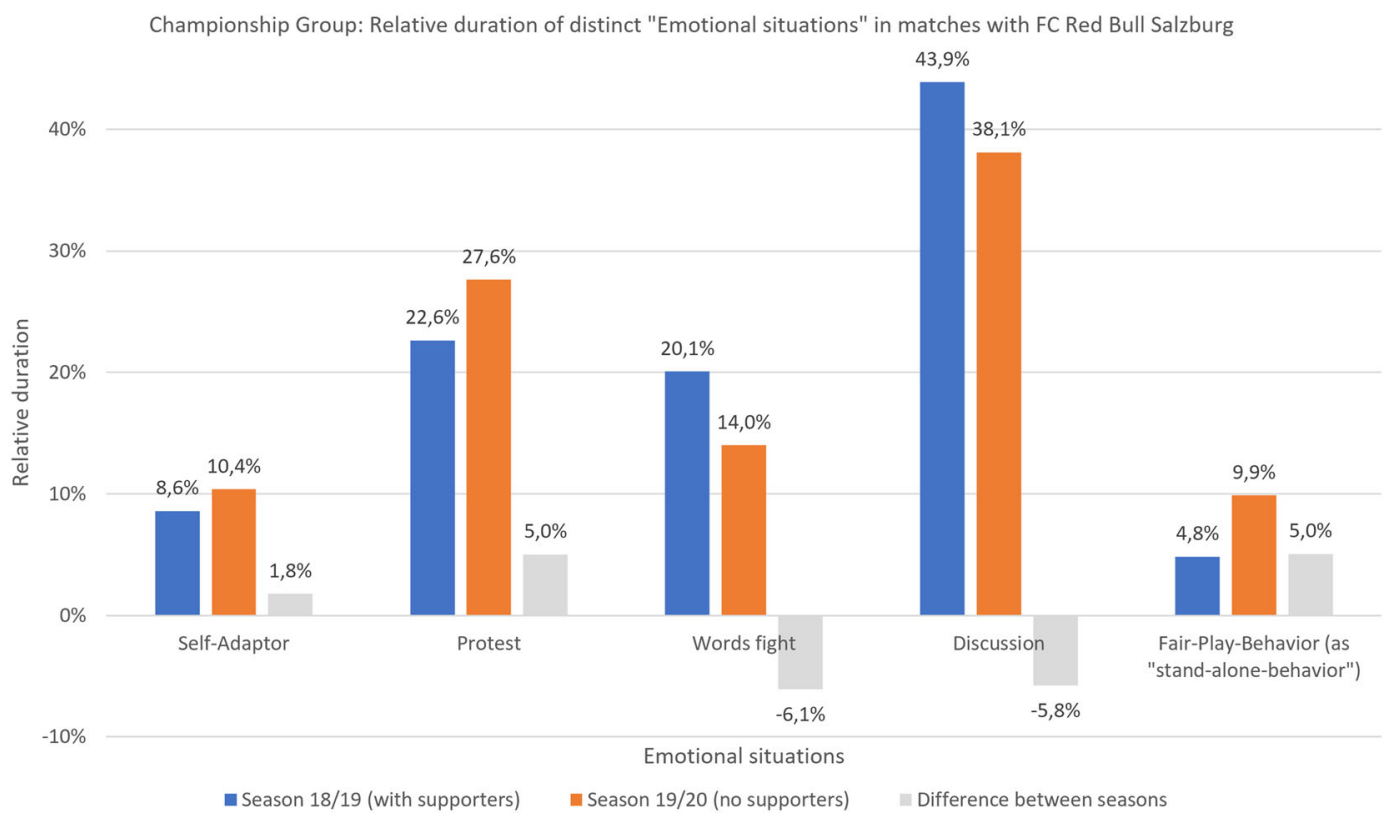

Fig. 4 Duration of "Emotional situations". Differences in the duration of distinct emotional behavior of players, staff, and officials in matches with FC Red Bull Salzburg (RBS) during the championship group phase in both seasons of 2018/19 and 2019/20 relative to the overall duration of emotional situations. Video documentation and analysis is based on our developed "Analysis System for Emotional Behavior in Football" (ASEB-F) (see above for details). Data shows the duration of emotional behavior "Self-Adaptor", "Protest", and "Fair-Play-Behavior" increased in "ghost games", whereas "Words fight" and "Discussion" decreased in "ghost games". This indicates that while the duration of players, staff and officials acting emotionally, predominantly decreased in "ghost games", "less extensive" behavior like "Self-Adaptor" and "Protest" increased in duration and "more extensive" behavior like "Words fight" and "Discussion" decreased in duration in "ghost games". Note that in this chart "Fair-Play-Behavior" was analyzed as "stand alone behavior", meaning that only "Fair-Play-Behavior" events were selected, which were not mixed up with any other emotional behavior (e.g., "Discussion" after foul, followed by "FairPlay-Behavior" between opponents). Results indicate that players showed twice as long "Fair-Play-Behavior" in "ghost games" than in regular matches with supporters. 
Spurny. Three Referees officiated only one game in one of the two seasons. These were Oliver Drachta, Gerhard Grobelnik and Markus Hameter in season 2018/19 and Walter Altmann, Manuel Schüttengruber and Felix Ouschan in season 2019/20. The age of the referees in the season without spectators (2019/20) shows that referees were, on average, 1.9 years younger than the referees of season 2018/19. Similarly, referees of season 2019/20 had their league debut in the Austrian Bundesliga earlier ( -26 months) and had less games $(-24)$ as head-referee than the referees of 2018/19. Although this could imply a "tilt for experience", the indices for mean cards per game (yellow, yellow-red and red cards) show no substantial differences between the two seasons: on average, referees of $2018 / 19$ and $2019 / 20$ differ by -0.020 yellow cards, 0.003 yellow-red cards and -0.031 red cards per game. This indicates that, although referees of season 2018/19 were on average older and (presumably) more experienced than referees of 2019/20, referees did not differ in booking players by means of showing authority on the pitch.

Our data from ASEB-F shows that in "ghost games" of season $19 / 20$, referees and other officials were less actively involved in emotional situations than in the same phase of season 18/19. In games with supporters (season 18/19), referees were actively involved in $39.4 \%$ of all documented emotional situations, whereas in games without supporters (season 19/20) referees were actively involved in only $25.2 \%$ of all documented emotional situations $(-14.2 \%)$, corresponding to a statistically significant difference and a large effect size $(t(18)=3.071,95 \%$ CI $[-1.517$, 8.083], $p=0.007, r=0.586$ ). From a temporal perspective (rounds 23 to 32) our data shows-except for rounds 27 and 32 -continuously higher numbers of emotional situations involving referees in RBS matches of season 18/19. In the same vein, the total number of emotional situations was higher in regular matches for season 18/19 (99 situations) than in "ghost games" for season 19/20 (51 situations). This leads to a total difference of $48.5 \%$ and (based on the different population of situations in 18/ 19 and 19/20) a relative difference of $14.2 \%$ fewer emotional situations documented involving referees in "ghost games". Results indicate that referees and officials acted less emotionally in "ghost games".

Further analysis shows that there are three categories of "emotional situations" in which referees were actively involved with players and/or staff. These are "Protest", "Words fight" and "Discussion". As illustrated in Fig. 5, comparison-by relative numbers-between season 2018/19 and 2019/20 shows that in "ghost games" referees were $-10.7 \%$ less involved in "Protests", $-9.7 \%$ less involved in "Words fight" and $-0.7 \%$ less involved in "Discussion" than in regular matches with supporters.

"Self-Adaptor", "Words fight", and "Fair-Play-Behavior". The comparison of players displaying "Self-Adaptor" in season 18/19 (39 situations) and 19/20 (33 situations), shows a relative difference of $0.8 \%$ more situations in "ghost games". From a chronological viewpoint-blocked in 15 min intervals of playing time-results show a sine wave akin development from kick-on to kick-off with obvious differences in relative magnitude in both seasons.

Comparison of players (and staff) displaying "Words fight" in season 18/19 (44 situations) and 19/20 (26 situations), shows a relative difference of $4.7 \%$ fewer situations in "ghost games". Chronological presentation shows a similar linear progression from kick-on to kick-off.

Comparison of players displaying "Fair-Play-Behavior" in season 18/19 (42 situations) and 19/20 (40 situations), shows a relative difference of $3.1 \%$ more situations in "ghost games". From a chronological viewpoint, players' behavior increases similarly in a linear way. A notable difference can be seen from minute 15 to 30 with strongly decreased "Fair-Play-Behavior" in games without supporters compared to games with supporters.

Owing to a similar chronological course we grouped "SelfAdaptor", "Words fight", and "Fair-Play-Behavior" together in Fig. 6. It is apparent that all three categories of documented behavior of both seasons-except early phases of "Fair-PlayBehavior" in matches of season 19/20 (no supporters)—follow a similar course over time.

"Protest" and "Discussion". "Protest" is displayed in 35.9\% (90 situations) of all documented situations in season 18/19 and in $40.1 \%$ (81 situations) of all documented situations in season 19/20. This means that in "ghost games" of RBS, $4.2 \%$ more "Protest" can be found in the behavior of players and staff (than in regular matches of 18/19). From a chronological viewpoint, an accumulation of "Protest" in games of season 19/20 (no supporters) is apparent from minute 15 to 30 compared to season 18/ 19 (with supporters).

"Discussion" is displayed in 19.9\% (50 situations) of all documented situations in season 18/19 and in 14.9\% (30 situations) of all documented situations in season 19/20. This means that in games of RBS, 5.1\% fewer "Discussion" can be found in the behavior of players and staff in "ghost games" of 19/20 than in regular matches of 18/19. Analysis from kick-on to kick-off shows that in games without supporters an accumulation of "Discussion" is apparent in the middle of the first half, whereas in games with supporters a similar accumulation of "Discussion" is evident in the beginning of the second half.

As illustrated in Fig. 7, both independent chronological courses of "Protest" and "Discussion" of season 19/20 (no supporters) develop similarly while courses of season $18 / 19$ (with supporters) develop differently from kick-on to kick-off.

\section{Discussion}

From a sport psychological perspective, basic game data such as "Committed Fouls" and overall "Disciplinary Points" appear similarly in regular games (with supporters) and "ghost games" (without supporters). This implies that the two championship group seasons of 2018/19 (supporters) and 2019/20 (no supporters) - with respect to players' behavior-are essentially comparable. Likewise, the league scenario for FC Red Bull Salzburg (RBS) was similar, while consistently remaining first in the league table, the club won the title in both seasons. Additionally, the club's lead in points and points lead development to the next ranked team was similar in both seasons.

Regarding players transfers between season 2018/19 and 2019/ 20 , our findings show that, although a substantial number of players left the club, the overall number of players getting involved in emotional situations in the following season was similar. This means that the number of "high involvement players" appeared stable in numbers and interactions and, therefore, no behavioral outliers biased the comparison of the two seasons. Although more fouls were committed in "ghost games", the difference in "Disciplinary Points" (which include bookings) are marginal. This also leads to the conclusion that-from a sportsmanship's perspective-RBS players behaved very similarly in both phases of the two seasons. All in all, there are reasonable grounds to assume that the basic prerequisite for two comparable seasons is given.

Comparison of the whole championship groups data of season 2018/19 and 2019/20 reveals that the league's trend to more scored goals (20\%) and fewer "Disciplinary Points" (-13.5\%) holds true. Therefore, we argue that the analyzed data of matches with RBS participation is representative for players', staff's and 


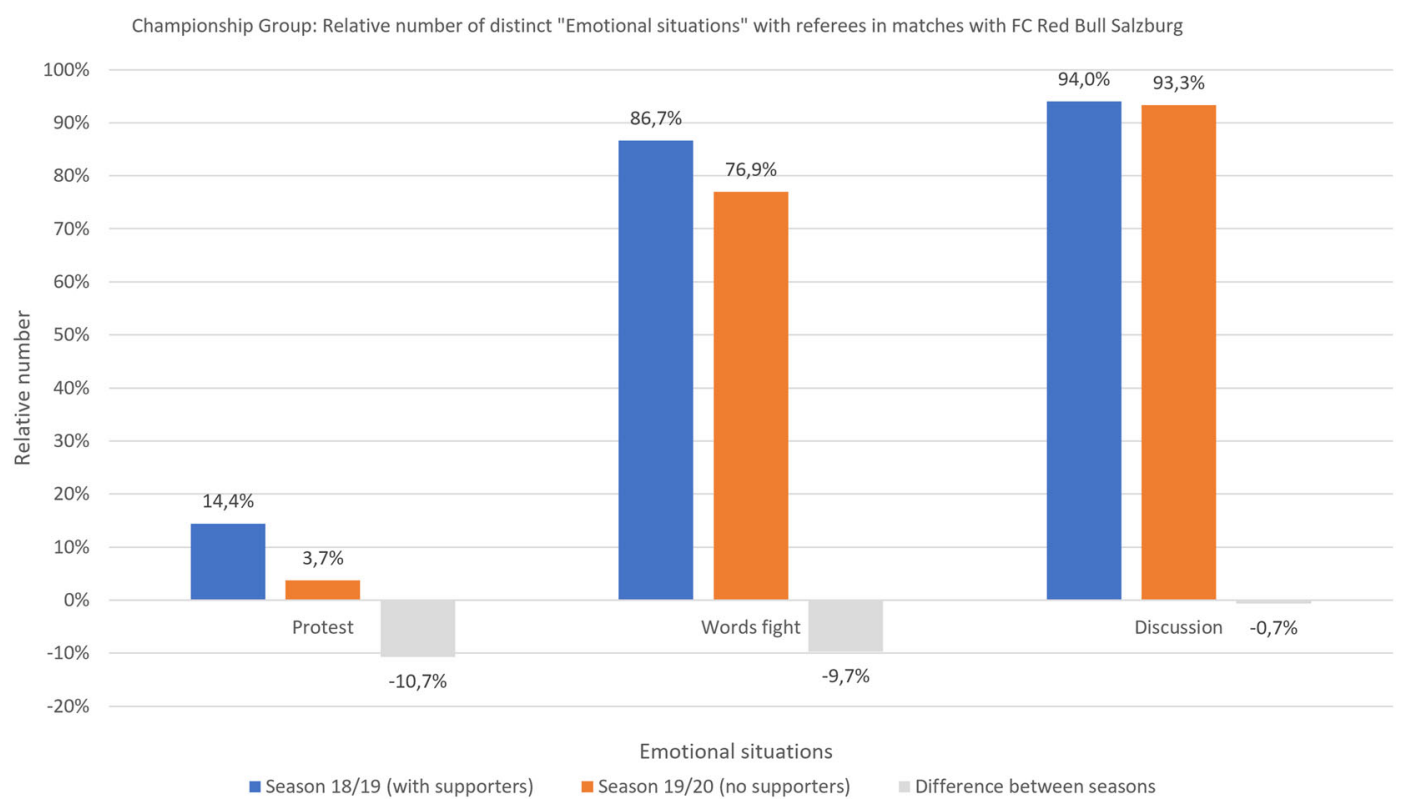

Fig. 5 Relative number of "Emotional situations" with referees. Differences in the relative number of distinct emotional behavior with officials involved in matches with FC Red Bull Salzburg (RBS) during the championship group phase in both seasons of 2018/19 and 2019/20. Video documentation and analysis is based on our developed "Analysis System for Emotional Behavior in Football" (ASEB-F) (see above for details). Data shows that the relative number of emotional behavior "Protest", "Words fight" and "Discussion" decreased in "ghost games". Thus, results indicate that officials acted less emotionally on players and staff behavior in "ghost games" than in regular games with supporters.

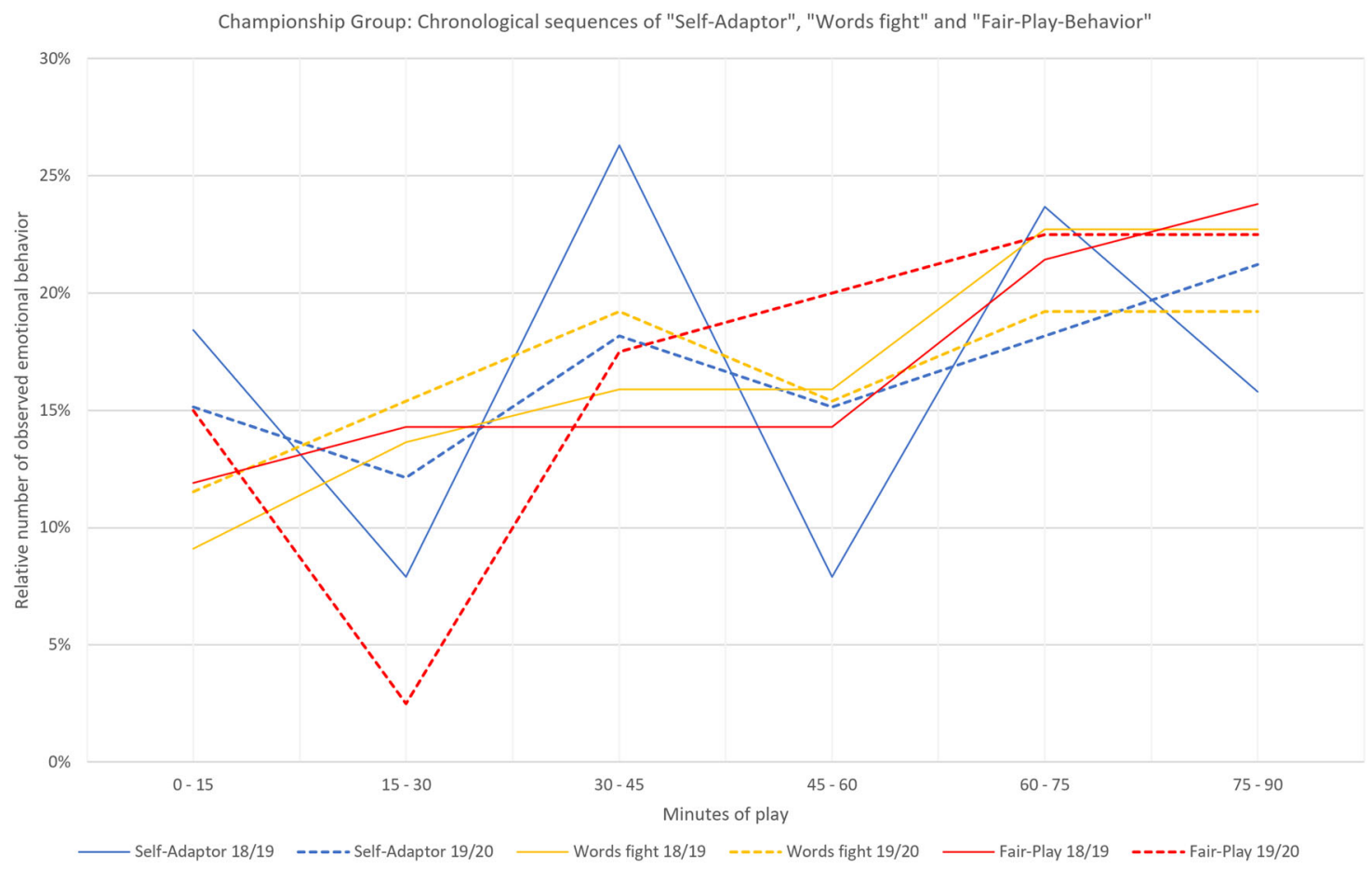

Fig. 6 Chronological sequences of "Self-Adaptor", "Words fight", and "Fair-Play-Behavior". Chronological development of relative numbers of players displaying "Self-Adaptor", "Words fight", and "Fair-Play-Behavior" in matches of FC Red Bull Salzburg (RBS) during the championship group phase in both seasons of 2018/19 (continuous lines) and 2019/20 (dotted lines). Graphical distribution illustrates a similar pattern for "Self-Adaptor" across seasons over time (continuous blue line: season 18/19 / dotted blue line: season 19/20), indicating that "ghost games" influence the number of observable "SelfAdaptor", but not the frequency of occurrence in the different phases of a professional football match. Graphical distribution illustrates a similar pattern for "Words fight" across seasons over time (continuous yellow line: season 18/19/ dotted yellow line: season 19/20), indicating that "ghost games" influence the number of observable "Words fight" in the first half-leading to more "Words fight" in "ghost games". In contrast, more "Words fight" can be documented in season 18/19 in the second half. Data further indicates that there is a constant chronological increase in "Words fight" in both seasons and that the effect of "ghost games" seems marginal and balances out over time. Regarding "Fair-Play-Behavior" a noticeable decline in "ghost games" of season 19/20 (dotted red line) in the mid of the first half is evident, followed by a subsequent rise, similar to changes in season 18/19 (continuous red line). 
$40 \%$

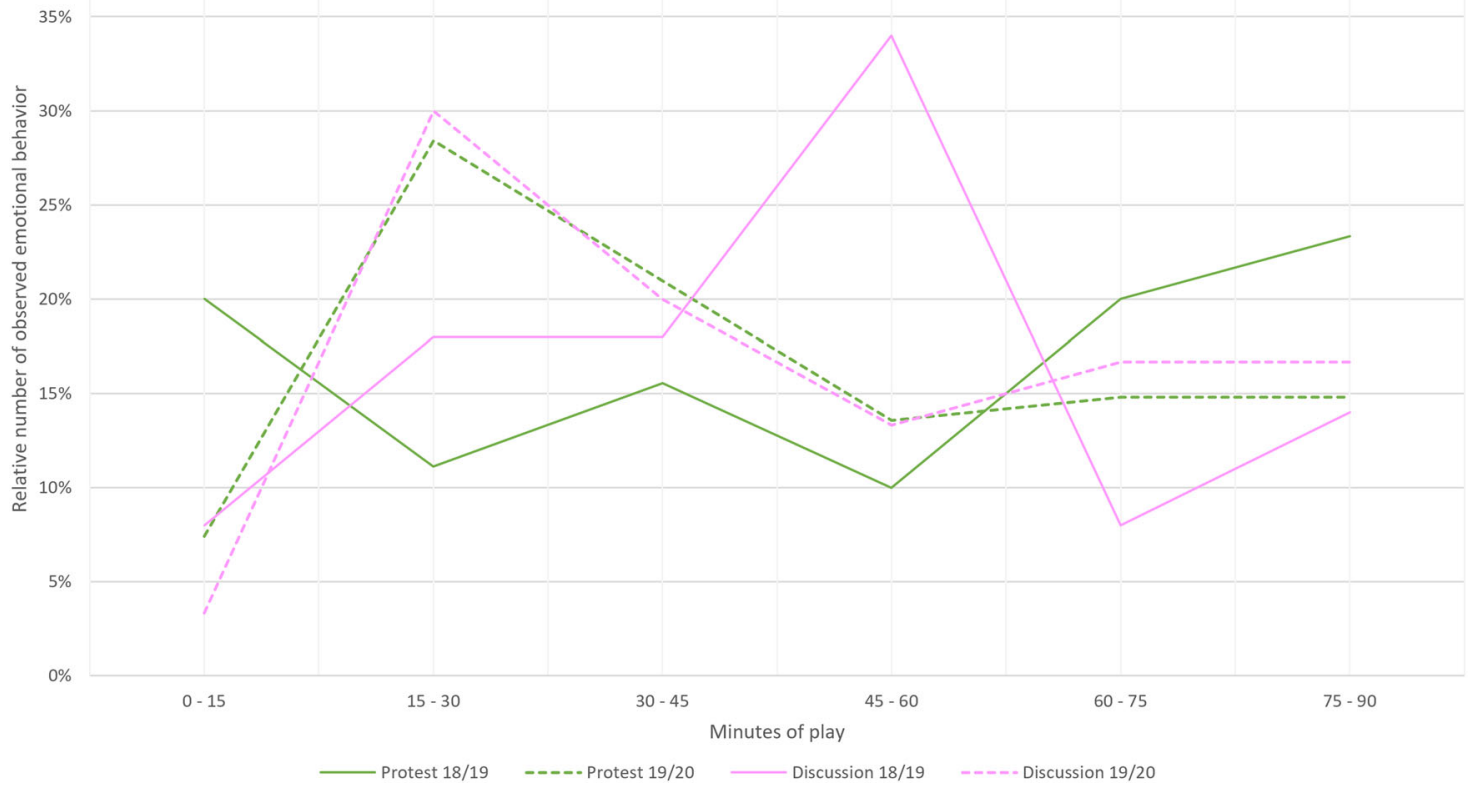

Fig. 7 Chronological sequences of "Protest" and "Discussion". Chronological development of relative numbers of players (and staff) displaying "Protest" in matches of FC Red Bull Salzburg (RBS) during the championship group phase in both seasons of 2018/19 (continuous green line) and 2019/20 (dotted green line). Graphical distribution illustrates a noticeable accumulation of "Protest" in "ghost games" in the mid of the first half, which declines during the rest of the match. Contrarily, another noticeable accumulation of "Protest" in regular games of season 18/19 can be found towards the end of matches. Regarding "Discussion" during the championship group phase in both seasons of 2018/19 (continuous pink line) and 2019/20 (dotted pink line) graphical distribution illustrates a noticeable accumulation of "Discussion" in "ghost games" in the mid of the first half, and a second noticeable accumulation of "Discussion" in regular games in the early phases of the second half. This double peak formation indicates a chronological shift in players and staff behavior in "ghost games".

officials' behavioral developments-which establish in response to the unique situation of "ghost games"-in the whole championship group.

The most apparent difference between the seasons of 2018/19 and 2019/20 is the number of "emotional situations", as documented by our "Analysis System for Emotional Behavior in Football" (ASEB-F), which was specifically developed for this study. Results show that in "ghost games" overall, 19.5\% fewer "emotional situations" could be identified. This leads to the conclusion that the missing crowd indeed had an impact on the emotions and behavior of players, staff, and officials. It seems that without the external factor of supporters, players and staff acted more factually and got less carried away with longer-lasting and extensive "Words fight" and "Discussion", which decreased, respectively, by 4.7 and $5.1 \%$. By contrast, less extensive protests increased by $4.2 \%$ in "ghost games". In a similar vein, the total duration of emotional situations was markedly longer in regular matches of season 18/19 (41:42 min) than in "ghost games" of season 19/20 (27:09 $\mathrm{min})$. This leads to an absolute difference of 14:33 min less documented time spent in "ghost games" on "SelfAdaptor", "Protest", "Words fight", "Discussion" or "Fair-PlayBehavior". Thus, results indicate that players and team staff behaved predominantly less emotionally in "ghost games" than in regular matches with supporters. To our knowledge, there is no study with a comparable scope and analysis system like ASEB-F. Therefore, we could not explicitly contrast our results with evidence from previous studies. We think that our new assessment tool will be a viable solution for the analysis of emotional behavior of football teams in future studies.

"Ghost games" also seem to have an impact on the behavior of officials (referees, assistant referees, and 4th officials). Official matchday statistics indicate that the behavior, by means of showing authority on the pitch (by booking players with cards), did not differ between seasons. Whereas our data-obtained with ASEB-F-shows that in "ghost games" officials were, in total $(-48.5 \%)$ and relative numbers $(-14.2 \%)$, less involved in emotional situations. Specifically, they were noticeably less involved when players or staff enacted in "Protest" or "Words fight".

We interpret this behavior as a consequence of missing supporters as an external factor making officials potentially more open to clarify decisions in order to calm down nascent emotions on the pitch but also in the stands. In "ghost games" officials feel less obligated to further clarify decisions and to react to "less extensive" behavior. This hypothesis is supported by the fact that the relative number of "Discussion" in both seasons was almost identical $(-0.7 \%$ less in "ghost games"). It seems that officials tried to avoid "unnecessary" interaction to ensure factual decision making and not getting drawn into unnecessary interaction with players and staff when their behavior was "less extensive" (as in "Protest" or "Words fight") but acted similarly as in regular games when "Discussion" developed or seemed necessary.

From a chronological perspective (kick-on to kick-off), our data reveals that "Self-Adaptor" and "Words fight" show comparable timelines in both seasons. Specifically, "Self-Adaptor" shows a similar temporal pattern but differs in the relative number of observable situations between the two seasons of 2018/ 19 and 2019/209. "Words fight" increases constantly in both seasons and shows similar relative numbers from kick-on to kickoff. In contrast, "Protest" in "ghost games" is characterized by a steep rise from kick-on to minutes 15 to 30 , whereas it decreases noticeably from a high initial value in the first $15 \mathrm{~min}$ in regular 
games with supporters. At the same time, behavior associated with "Fair-Play" decreases in "ghost games" especially in the middle of the first half, while approximating later to levels observable in regular matches with supporters until kick-off. Time courses of "Discussion" show two separate peaks of behavior; the first rise in the middle of the first half in "ghost games" but not in regular matches, the second rise in the beginning of the second half in regular games but not in "ghost games", resulting in a double peak formation showing a chronological shift in players' and staff behavior.

Furthermore, our findings indicate that players and staff experienced especially the first $30 \mathrm{~min}$ of "ghost games" differently than those of regular matches. Particularly, players and staff display a high number of "Protest" in the middle of the first half while showing almost no "Fair-Play-Behavior", combined with a high number of "Discussion" with opponents and officials. Another major difference in the experience of players and staff can be found in the beginning of the second half (minutes 45 to 60) when "Discussion" rises noticeably in regular games with supporters but decrease in "ghost games".

A recent investigation of the International Centre for Sports Studies (CIES) Football Observatory suggests that matches without attendance had a significant impact on the magnitude of the home advantage in the Austrian Bundesliga ("CIES. Football Observatory. What about the home advantage," 2020). One might speculate that in the "ghost games" the arousal of the players of the home team was unusually low, leading to a worse performance of the home team. In contrast, the arousal of the players of the away team was just right, with highly activating factors such as playing in an unfamiliar and latently hostile environment still present, leading to a better performance of the away team. This interpretation, however, cannot be investigated with the present data and, therefore, other explanations for the home (dis) advantage in the "ghost games" cannot be ruled out. A more thorough investigation of the home (dis)advantage is beyond the scope of the present paper, but will be subject to analysis in a future paper including data from all major European football leagues.

Limitations. Owing to limited resources, we concentrated on the matches of one football club that we considered-from a sport psychological perspective-as stable and constantly thriving, leading to comparable data over two seasons. Although data analysis indicates that the documented behavior of players is representative for the whole championship group, we plan to further analyze other teams in detail to underpin this claim. As football games constitute complex social processes and interactions between various stakeholders, it is impossible to rule out every confounding variable. This is especially true when it comes to the comparison of different seasons due to matches without supporters because of a worldwide pandemic. We did our best to control for every possible factor and to explain sufficiently every methodological approach we implemented. We can, however, not rule out that there are variables we did not control for and, most likely, ultimately cannot be controlled for at all. Nonetheless, we believe that by publishing our methodology of ASEB-F and presenting our results in this case study, we can provide a novel approach and perspective on the investigation of emotional behavior on the pitch in general and of psychological effects of supporters in professional football in particular. This will help to further strengthen the reliability and validity of our developed approach.

It is reasonable to assume that a team's tactical formation has direct effects on the "role" every player is assigned to fulfill during the match. It is further conceivable that, based on tactical instructions, players feel more or less comfortable with their role, which could in return have an effect on how players react in certain emotional situations. For example, an offensive player, whose tactical role is to make small fouls-after the team loses the ball, to prevent the opposing team from counter-attackingprobably will choose to avoid getting booked too early in the game. To achieve this, a potential strategy is discussing friendly with the referee after every foul in order to downplay the situation. This is a decision a player makes based on his/her personality, motivation, mindset or daily state of mind and body and so potentially biases the analysis with ASEB-F.

In the same vein, another potentially biasing factor could be the fact that RBS had to find a new head coach after Marco Rose left the club in the summer between season 2018/19 and 2019/20. While Rose regularly let the team play 4-4-2 (diamond) and 4-23-1 formations, the new head coach Jesse Marsch preferably fields standard 4-4-2. This tactical shift probably produces changes in the roles of certain players. Owing to the complexity that comes with the analysis of natural interpersonal situations in social contexts, we believe that a certain degree of potential bias is part of the nature of behavioral observations on the pitch and impossible to compensate for completely.

Furthermore, based on our data we cannot clarify whether officials' reduced involvement in emotional situations in "ghost games" can be ascribed to players and staff behavior or to referees feeling less obliged or less willing to react to players and staff differences of opinion. We plan to further investigate this question with a follow-up study on the experiences and observations of officials in "ghost games".

\section{Conclusions}

"Same same but different"-from a sport psychological perspective we argue that the findings of our "ghost games" study indicates that the absence of supporters has a substantial influence on the experience and behavior of players, staff and officials alike.

\section{Data availability}

The datasets generated and/or analyzed during the study are not publicly available due to legal reasons but are available from the corresponding author on reasonable request.

Received: 10 August 2020; Accepted: 14 December 2020; Published online: 26 January 2021

\section{References}

Allport FH (1924) Soc Psychol. Houghton Mifflin, Boston

Baumeister RF (1984) Choking under pressure: self-consciousness and paradoxical effects of incentives on skillful performance. J Pers Soc Psychol 46 (3):610-620

Carron AV, Loughhead TM, Bray SR (2005) The home advantage in sport competitions: Courneya and Carron's (1992) conceptual framework a decade later. J Sports Sci 23(4):395-407

CIES (2020) Football Observatory. What about the home advantage after the COVID-19 pandemic? https://football-observatory.com/What-about-thehome-advantage-after-the-COVID-19-2470. Accessed 11 Nov 2020

DerStandard (2020) Bundesliga-Trainer einig: Lust auf Fußball größer als CoronaBedenken. https://www.derstandard.at/story/2000117697659/bundesligatrainer-einig-lust-auf-fussball-groesser-als-corona-bedenken. Accessed 20 Jul 2020

DerStandard (2020) Sportpsychologe über Geisterspiele: "Resonanzloser Raum”. https://www.derstandard.at/story/2000117722891/sportpsychologe-uebergeisterspiele-resonanzloser-raum. Accessed 20 July 2020

Downward P, Jones M (2007) Effects of crowd size on referee decisions: analysis of the FA Cup. J Sport Sci 25(14):1541-1545

Eaves MH, Leathers D (2018) Successful nonverbal communication. Principles and applications, 5th edn. Taylor \& Francis Group, New York and London 
Lazarus RS (2000) How emotions influence performance in competitive sports. Sport Psychol 14:229-252

Nevill AM, Balmer NJ, Williams AM (2002) The influence of crowd noise and experience upon refereeing decisions in football. Psychol Sport Exerc 3:261-272

Pettersson-Lidbom P, Priks M (2010) Behavior under social pressure: empty Italian stadiums and referee bias. Econ Lett 108:212-214

RAN (2020) Fußball: Die Restart-Termine der europäischen Ligen nach der Corona-Pandemie. https://www.ran.de/fussball/international/bildergalerien/ fussball-die-restart-termine-der-europaeischen-ligen-nach-der-coronapandemie. Accessed 20 Jul 2020

Unkelbach C, Memmert D (2010) Crowd noise as a cue in referee decisions contributes to the home advantage. J Sport Exercise Psy 32:483-498

Varca PE (1980) An analysis of home and away game performance of male college basketball teams. J Sport Exerc Psychol 2(3):245-257

Wagstaff CRD (2014) Emotion Regulation and Sport Performance. J Sport Exercise Psy 36:401-412

Zajonc RB (1965) Social facilitation. Science 149:269-274

\section{Acknowledgements}

We would like to thank the following people, to whom we are profoundly grateful, for supporting us and without their help this study would not have been possible: Uwe König (Sky Austria)/Wolfgang Skarich (Sky Austria)/Matthias Wiederin (Stats Perform)/ Christoph Glasner (FC Red Bull Salzburg)/Patrick Kastner (Study Assistant)/Isobel Sophie Klier (Scientific Secretariat)

\section{Competing interests}

The authors declare no competing interests.

\section{Additional information}

Correspondence and requests for materials should be addressed to M.C.L. or F.R.

Reprints and permission information is available at http://www.nature.com/reprints

Publisher's note Springer Nature remains neutral with regard to jurisdictional claims in published maps and institutional affiliations.

(c) (i) Open Access This article is licensed under a Creative Commons Attribution 4.0 International License, which permits use, sharing, adaptation, distribution and reproduction in any medium or format, as long as you give appropriate credit to the original author(s) and the source, provide a link to the Creative Commons license, and indicate if changes were made. The images or other third party material in this article are included in the article's Creative Commons license, unless indicated otherwise in a credit line to the material. If material is not included in the article's Creative Commons license and your intended use is not permitted by statutory regulation or exceeds the permitted use, you will need to obtain permission directly from the copyright holder. To view a copy of this license, visit http://creativecommons.org/ licenses/by/4.0/.

(c) The Author(s) 2021 